\title{
Studies of Extended Southern Galactic Clusters and Loose Star Groupings using the Bosscha Schmidt Telescope
}

\author{
S. D. Wiramihardja, B. Hidayat, I. Tejawijaya, Hakim, L. Malasan \\ Bosscha Observatory and Department of Astronomy, Bandung Institute \\ of Technology, Bandung 40391, Indonesia
}

\section{Introduction}

One of the programs conducted with the Bosscha Schmidt telescope is the study of loose star groupings and extended galactic clusters in the southern hemisphere (The 1963; Hidayat \& Wiramihardja 1978; Hidayat et al. 1994). The aims of the study are, in short, to :

1. investigate or to confirm the physical reality of the loose groupings.

2. study the relationship between extended clusters or loose groupings with their environment.

3. derive their physical parameters which ultimately can be used to construct the luminosity functions of the objects.

The purpose of the present paper is to present one of our results for the extended cluster OCL 1104-584 (NGC 3532). The results of studies of loosegrouping objects taken from the list of Loden (1979) will be presented elsewhere.

\section{Observations}

All the photometric data were collected with the Bosscha Schmidt telescope with the plate-filter combination of $1 \mathrm{IaO}+\mathrm{GG} 13$ for $B$, and $103 \mathrm{aD}+\mathrm{GG} 11$ for $V$. In all cases we secured spectral data on IIaO or $103 \mathrm{aO}$ plates exposed without any filter. The exposure times range from 2 to 5 mins for direct plates and from 10 to 60 mins for spectral plates. The $6^{\circ}$ objective-prism attached to the Schmidt telescope yields a dispersion of $315 \AA \mathrm{mm}^{-1}$ at $\mathrm{H} \gamma$ on our spectral plates.

\section{Photographic Photometry}

Photographic photometry of the program stars was derived using the photoelectric magnitudes of standard stars for calibration. The plates were measured with an Eichner astrophotometer of the Bosscha Observatory, or using the Nalumi microdensitometer. In some cases the plates are calibrated with a wedge sensitometer. 


\section{Method}

One of the tools used to search for new star clusters is to identify apparent stellar groupings on objective-prism spectral plates. When some stars with almost the same brightness show similar type of spectra in a limited volume of space one would suspect that they form a real grouping. However, it might be possible that some of them are not members. On the other hand, there is a possibility that there are other fainter member stars which are too faint to be detected by spectral surveys. Photometric study should be able to resolve whether the suspected cluster is real or not and to investigate other fainter members. Observations with a wide field telescope help to delineate the possible extended area of the groupings.

An indication which favours the physical existence of a cluster is the apparent concentration of stars. Therefore, we first define the boundary of the stellar concentration around the suspected cluster by comparing the stellar density distribution within the boundary with that of the field density. The second step is to construct the colour-magnitude diagram of program stars upon which the standard ZAMS curve can be fitted. The fitting is done by shifting the ZAMS curve with the adopted value of colour excess, derived from the interrelation between colour excesses and distances of some other clusters and stars found in the general direction of the suspected cluster. The membership of the cluster can be derived by inspecting the location of program stars along the ZAMS. The confirmation of membership could be done using a $V_{0} / M_{0}$ diagram (Walker $1965)$ and an evolutionary deviation curve. We employ the calibration of Meynet et al. (1993) to obtain the best isochrones. The distance of the cluster is derived by using the adopted value of colour excess and the distance modulus of the cluster estimated from its colour magnitude diagram.

Wherever possible, data on radial velocities and proper motion of stars within and around the suspected cluster are incorporated in the analysis. For our future studies of NGC 3532 the proper motion data of King (1978) will be included in the analysis.

\section{The Cluster NGC 3532}

This cluster is a rich grouping of stars, located against a rather extensive and complicated galactic background. Some of the photometric properties of stars in the cluster have been studied by Wizinowich \& Garrison (1982). The authors noted, after comparison with photometric results obtained earlier, several differences of the photometric results. We confirmed the discrepancies detected by them.

In order to ensure uniform magnitude and colour determination for NGC 3532 and the neighbouring stars, background corrections were applied for all sequence stars in the neighbouring clusters. Two hundreds and eighty nine stars in and around NGC 3532 were used for our study. The local magnitudes and colours are tied with the UBV system by using the photoelectric sequence established in NGC 3572.

Our study of interstellar reddening, based on stars with MK spectral types found in the general direction of the field NGC 3532 and 3572 , reached a value of 


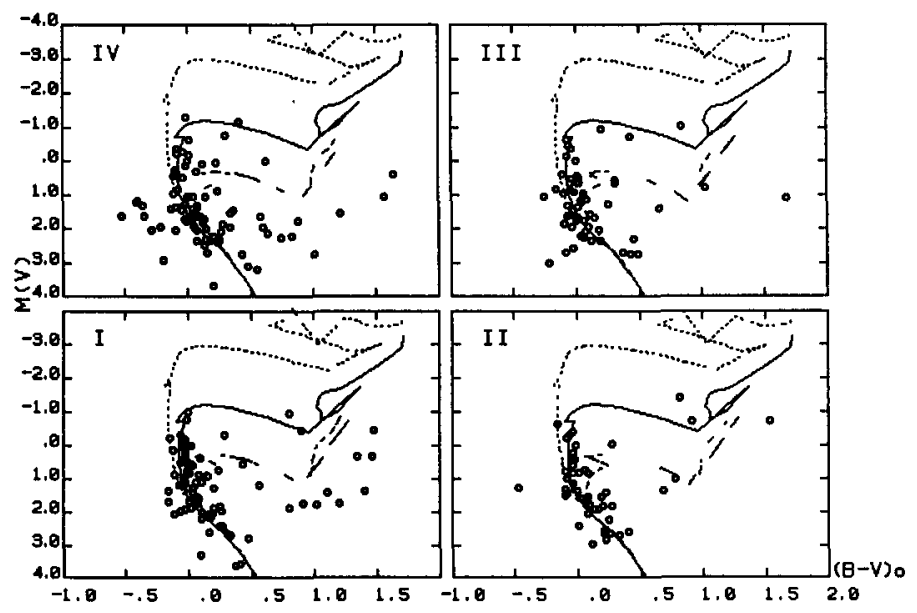

Figure 1. Observed C-M diagram for each quadrant of NGC 3532. Theoretical C-M diagrams corresponding to $\log t=8.0,8.5$, and 9.0 are given by the dotted line, solid line and dashed line, respectively.

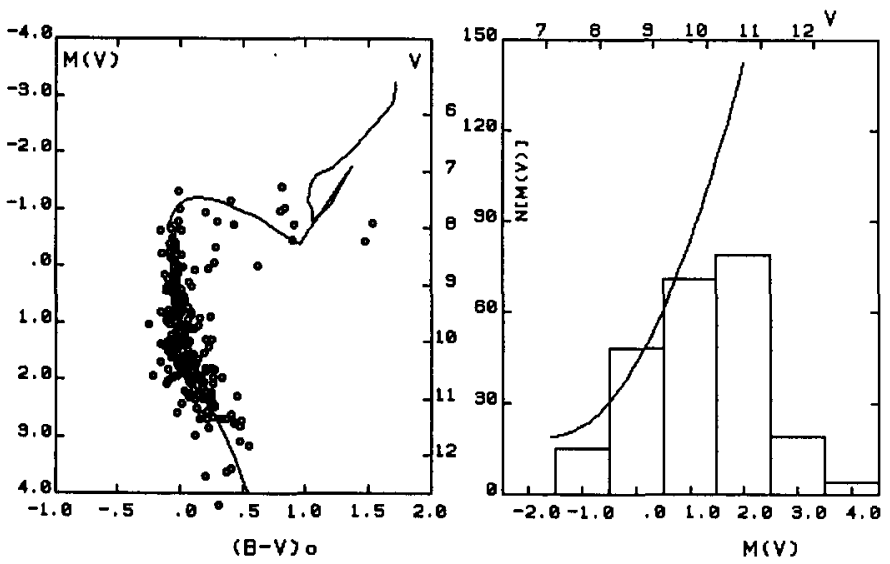

Figure 2. Theoretical C-M diagram for $\log t=8.5$ superposed on the observed one which contains photometric probable members (left), and the observed luminosity function of NGC 3532 (right). The solid line represents Taff's average value of the luminosity function for rich clusters. 
0.2 mag. Battinelli et al. (1994) quoted a reddening of 0.04 mag. Using $\mathrm{E}_{B-V}=$ $0.02 \mathrm{mag}$, the distance modulus of 8.57 can be assigned for the cluster by fitting the observed C-M diagram with the standard main sequence for normal stars. The distance modulus corresponds to the true distance of $529 \mathrm{pc}$. In order to check the result we have studied the evolutionary deviation curve. There is no significant difference between the two results.

Stellar counts have been performed for two reasons. One is for the determination of the cluster diameter. Based on star fields of 15 arcmin $\times 15$ arcmin and using various magnitude limits (from 12 through $14 \mathrm{mag}$ ) we obtained the diameter of $50 \mathrm{arcmin}$. This value is adopted in the analysis of the luminosity function of the cluster. The second reason for performing the stellar counts was to derive the distance of the dark cloud which is found in the general direction of NGC 3532 and NGC 3572. Using the Wolf method and employing stars of absolute magnitude -4.95 , we obtained a distance of $3 \mathrm{kpc}$ for the cloud. In view of the great difference in distance between the cloud and the clusters, there seems to be no spatial connection between the dark nebulae and the cluster complex.

NGC 3532 is divided into 4 quadrants, each containing about the same number of stars. The C-M for each quadrant is constructed. Fig. 1 shows the observed C-M diagram for each quadrant superposed with the theoretical C-M diagram of various isochrones taken from Meynet et al. (1993). Although a small spread can be observed, the fitting of the C-M diagram suggested that $\log t=$ 8.5 is the best fit. Thus $t=10^{8.5} \mathrm{yr}$ is adopted as the age of the cluster. It would be instructive to study in detail the character of its luminosity function as a rich cluster of intermediate age. This is presented in Fig. 2, where the Taff (1974) average value of the luminosity function (solid line) is superposed. The luminosity function indicates that the maximum is reached at $M=12$, (corresponding to $V=10.5$ ) and then declines sharply presumably due to selection effects. The slope between $M_{V}=-2.0$ to $M_{V}=+2.0$ is similar to that of Taff; which is rather surprising in particular if one compares with that of NGC 5662 (Claria et al. 1991). The cluster has similar richness of stars but is slightly different in age.

\section{Acknowledgements}

We acknowledge with many thanks the support from the Warner and Swasey Observatory (1979-1980) and from the LKBF (1984-1985) for plates used in this study.

\section{References}

Battinelli P., Brandimarti A. \& Capuzzo-Dolcetta R. 1994, A\&AS, 104, 379

Claria J. J. \& Lapasset E., 1988, MNRAS, 235, 1129

Hidayat B., Malasan H. L., Wiramihardja S. D. \& Sukardi H. R. 1994, in progress

Hidayat B. \& Wiramihardja S. D., 1978, A\&AS, 34, 73

King D. D., 1978, J. Proc. R.S.N.S.W, 3, 1 
Loden L. O., 1979, A\&AS, 36, 83

Meynet G., Mermilliod J. C. \& Maeder A. 1993, A\&AS, 198, 477

Taff L. G., 1974, AJ, 79, 1280

The P. S., 1963, Bosscha Contribution, 32

Walker G. A. H., 1963, ApJ, 141, 660

Wizinowich P. \& Garrison R. F. 1982, AJ, 87, 1390 